\title{
Pour les institutions religieuses, la communication est devenue un véritable outil de gestion
}

\section{Bernard Dagenais}

\section{(2) OpenEdition \\ 12 Journals}

Édition électronique

URL : http://journals.openedition.org/communicationorganisation/1834

DOI : 10.4000/communicationorganisation. 1834

ISSN : 1775-3546

Éditeur

Presses universitaires de Bordeaux

\section{Édition imprimée}

Date de publication : 1 mai 1996

ISSN : 1168-5549

Référence électronique

Bernard Dagenais, «Pour les institutions religieuses, la communication est devenue un véritable outil de gestion », Communication et organisation [En ligne], 9 | 1996, mis en ligne le 26 mars 2012, consulté le 21 septembre 2020. URL : http://journals.openedition.org/communicationorganisation/1834 ; DOI https://doi.org/10.4000/communicationorganisation.1834

Ce document a été généré automatiquement le 21 septembre 2020.

(c) Presses universitaires de Bordeaux 


\title{
Pour les institutions religieuses, la communication est devenue un véritable outil de gestion
}

\author{
Bernard Dagenais
}

1 «Dieu n'a pas besoin de marketing, écrivait Flipo (1984), mais l'Église si ». Flipo errait, car aujourd'hui, l'un et l'autre doivent affirmer leur existence au milieu des nouvelles réalités religieuses.

2 Lorsque les grandes religions étaient confinées dans une aire géographique donnée, il leur était facile d'affirmer que le vrai Dieu était le leur. Et quand, de ces aires géographiques, se préparaient des conquêtes, on transportait et imposait le vrai Dieu. Aujourd'hui, dans de multiples pays, les différentes religions se côtoient avec ou sans discrimination: catholique, protestante, islamique, juive, hindoue. Dans chaque religion, des écoles de pensée s'affrontent. Et à côté d'elles se développe une nouvelle spiritualité incarnée par les sectes multiples, les gourous tout puissants, les mouvements holistiques, messianiques ou apocalyptiques.

Et chacun revendique toujours la primauté de son Dieu et la sagesse suprême de "sa » religion. Dans une exposition sur la mort tenue au printemps 1996, le Musée de la civilisation du Québec, présentait ainsi l'existence des dieux de la terre :

«L'Islam est issu du judaïsme et offre une croyance en un salut auprès d'un Dieu, Allah.

5 Issu du judaïsme, le christianisme offre une croyance en un salut auprès de Dieu qui pardonne et accueille les morts auprès de lui, pour y vivre une vie nouvelle et éternelle. Le judaïsme offre une croyance en un salut auprès d'un Dieu, Yahvé ».

7 Pour Frossard, Dieu existe, il l'a rencontré. Le héros de La douane de mer de D'Ormesson a attendu quelques jours après sa mort avant de se présenter devant le seigneur. Pivot demande à chacun de ses invités ce qu'ils aimeraient entendre Dieu leur dire au moment de leur mort. Nous sommes loin de La divine comédie de Dante ou le ciel était plus complexe que le rapport direct à Dieu. 
8 Aujourd'hui les guerres de religion se perpétuent encore dans certaines parties du globe. Le fanatisme religieux n'a jamais cessé d'exister. On tue en Algérie au nom de l'intégrisme islamique. On s'entretue en Inde autour de la symbolique d'un lieu du culte. On tue en Bosnie au nom d'un idéal ethnique qui recoupe des différences de religion. Et on tue aux États-Unis au nom du christianisme (le cas des cliniques d'avortement). Au cours des dernières années, « 34 églises de paroisses noires dans le sud des États-Unis sont parties en fumée, bien que le Conseil national des Églises estime que ce sont plus de 50 lieux de culte qui ont été incendiés volontairement depuis 1990 ” (Sanz 1996). On se bat devant les tribunaux en Allemagne pour savoir si les crucifix peuvent être accrochés au mur des écoles (Jackson 1995).

9 Au nom de la tolérance, les Sikhs du Canada obtiennent le droit de porter, au sein de la Gendarmerie Royale du Canada, le turban au lieu du célèbre chapeau de la police montée. En Amérique latine, la visite du pape au début de 1996 a démontré la force, la vigueur et la détermination des églises "évangéliques». Au Guatemala, celles-ci qualifiaient la venue du pape comme l'arrivée de l'Antéchrist. Et les catholiques répondaient que les évangélistes constituaient l'opium du peuple et qu'ils étaient au service de pouvoirs étrangers (Serrill 1996).

10 Aujourd'hui, le terme même de religion couvre un vaste horizon. Après avoir dressé une frontière entre religion et magie chez les peuples sans écriture, on hésite aujourd'hui à regrouper sous un seul vocable l'univers de la spiritualité. Car la spiritualité peut exister sans Dieu et Dieu sans spiritualité. Et la croyance en un Dieu peut s'exprimer à l'extérieur de la religion ; tout comme la pratique de la religion peut s'effectuer sans véritable croyance.

11 Avec la baisse de la pratique religieuse dans de nombreux pays développés, avec les difficultés de recrutement de nouveaux membres, avec, en parallèle, la montée spectaculaire de l'idéal religieux sous toutes ses formes, que l'on qualifie d'intégrisme chez les uns ou de conviction profonde chez les autres, Dieu, les dieux, les religions, les sectes, les formes d'attitude spirituelles n'ont jamais tant eu besoin de communiquer, d'affirmer sinon d'imposer leur existence.

12 Si l'acculturation religieuse se pratique toujours autour d'une dynamique interpersonnelle, si la réalité financière de ces empires se construit par l'implication personnelle des fidèles, l'existence sociale, légale et morale de ces institutions passe par la communication publique. Comme de véritables partenaires socio-politiques, elles n'hésitent pas à appuyer des causes, à soutenir des points de vue et à affirmer des idées. Désormais, il ne s'agit plus uniquement de prêcher la bonne parole et de sauver les âmes individuelles, toute église veut de plus reconquérir son statut de partenaire social privilégié et affirmer son pouvoir sur le processus qui entoure toute décision. La religion se repolitise... Et pour ce faire, elle doit accepter d'entrer dans l'arène sociale, politique et médiatique.

14 La communication est devenue un outil essentiel de gestion pour la religion comme pour toute institution. Tout comme la politique, l'économique, le culturel et le social se définissent dans et pour les médias, l'institution religieuse ne peut plus échapper à l'attrait de la médiatisation. Les voyages du pape à l'étranger constituent de véritables événements médiatiques. Les télévangélistes américains ont élevé la religion au rang de spectacle. L'intégrisme islamique utilise le terrorisme comme forme de discours. Et les multiples sectes orchestrent leur devenir par des stratégies de communication bien 
structurées : soit elles construisent un mur de silence bien opaques autour d'elles ; soit elles exploitent leur raison d'être pour acquérir plus de renommée : autant l'Ordre du Temple Solaire a choisi de vivre replié sur lui-même, autant par exemple, le Parti de la loi naturelle, qui prône la méditation transcendale, fait feu de tout bois pour faire parler de lui.

Que ce soit à l'interne pour conserver la cohésion du groupe et ancrer les préceptes de l'institution ou à l'externe pour attirer de nouveaux adeptes et recueillir les fonds nécessaires pour survivre et se développer, les institutions religieuses ont appris à gérer leurs communications. Ainsi, pour attirer des fidèles, les Églises ont recours à la publicité (Cleland 1995). Pour recueillir des fonds, elles confient à des agences spécialisées leur stratégie. Pour évaluer l'importance de leurs compétiteurs, elles initient des recherches (Gerbner et al, 1984). Pour connaitre le pouls de leurs ouailles, elles distribuent des sondages et reçoivent les appels téléphoniques (Baillargeon 1996). Et elles n'hésitent surtout pas à faire connaître ces démarches par des activités de relations publiques : conférence de presse, entrevue, grand rassemblement.

Par ailleurs, la richesse, les angoisses et les déceptions de l'âme humaine demeurent une préoccupation continue. Cette fin du XIX ${ }^{\mathrm{e}}$ siècle est celui de la maladie de l'âme, le retour en force sur les interrogations métaphysiques que provoque une société trop matérialiste.

17 Tout contribue à faire de la religion un partenaire socio-politique important, sinon essentiel de la société humaine. Et pourtant, la religion n'arrive pas à faire bon ménage avec les médias. Selon les intervenants qui se sont penchés sur cette question, deux problèmes se posent : les médias détruisent les valeurs profondes de l'être humain; et les médias n'accordent pas assez d'attention à la religion.

Le célèbre auteur américain John Updike (1996) a choisi, pour son 17ième roman, In the Beauty of the Lilies, de s'interroger sur la coïncidence du déclin de la foi religieuse avec la montée du cinéma. C'est toujours la faute aux médias.

19 Le Freedom Forum Media Studies Center organisa en 1993 un colloque sur les rapports entre religion et médias. De façon presque unanime, les participants ont manifesté leur grande déception face à l'attitude des médias qui semblaient nier à la religion sa présence sociale. Cette attitude s'exprimait de multiples façons.

20 D'abord, il y a peu de spécialistes de la religion dans les médias. Si l'on compare la religion avec le sport, la culture et l'économie par exemple, on constate qu'il y a plus de gens qui assistent chaque semaine à des manifestations religieuses qu'à des manifestations sportives, culturelles ou qui participent à des activités économiques. Or, le poids des chroniqueurs spécialisés de ces différents secteurs écrase, dans chaque média, celui du chroniqueur religieux, lorsqu'il y en a un.

21 Le premier corollaire de cette décision éditoriale s'impose : l'espace consacré à la religion est infime par rapport à l'importance sociale réelle de cette institution. En fait, sauf exception, pour que la religion attire l'attention des médias, il faut une nouvelle vraiment hors du commun (Dennis 1993).

Le second corollaire confirme le peu de cas que les médias accordent à la religion. Ceuxci manifestent une réticence certaine à inclure un éclairage religieux dans l'analyse des grands enjeux sociaux. Lors des crises, des controverses, des décisions importantes pour la société, les médias ont recours à des spécialistes pour jeter un peu de lumière sur ces phénomènes : universitaires, économistes, politiciens, syndicalistes, tous sont 
appelés à se prononcer sur les grands enjeux de la société. Jamais on n'intègre le volet religieux à ces analyses. Or, très souvent, les décisions ont une dimension morale certaine, morale issue de la foi même des individus. Les guerres de libération sont discutées par les personnalités politiques, le suicide assisté par les médecins, les avocats et les criminologues, la détresse humaine par les groupes caritatifs. On n'interroge jamais le monde religieux sur ces sujets.

Selon certains spécialistes qui ont participé au colloque de Freedom Forum, il existe dans les salles de rédaction et de nouvelles une certaine culture de scepticisme et d'ignorance face à la religion, et dans certains cas, une hostilité non déguisée. Une enquête réalisée par Wyatt a toutefois démontré que les médias sont plus indifférents qu'hostiles à la religion (Dart et Allen 1993, p. 4).

Certains journalistes et producteurs de télévision assurent que la séparation de l'Église et de l'État rend inappropriée toute intervention de la religion dans les débats politiques (Dart et Allen, 1993, p. 12). Or, selon certains observateurs, tout journaliste devrait inclure la dimension religieuse dans sa perception de la réalité. Comment peuton comprendre ce qui se passe au Moyen-Orient, en Inde, en ex-Yougoslavie, si on n'y inclut pas la dimension religieuse? Comment interpréter les changements dans les valeurs fondamentales nord-américaines, si on exclut la religion, la moralité et l'éthique ? Comment saisir la véritable portée des découvertes sur l'origine du monde, si on ne les intègre pas aux enseignements de la religion?

25 Enfin, les thèmes retenus et l'angle que choisissent les médias pour traiter de la religion, en s'attardant sur le spectaculaire et la controverse, donnent une image déformée de la réalité religieuse.

\section{Les grandes lignes de ce numéro}

Ce numéro de la revue Communication \& organisation veut se pencher sur l'étude des différentes stratégies de communication utilisées par les institutions religieuses pour affirmer leur existence. Conscientes des difficultés qu'elles ont d'obtenir dans les médias la place qu'elles estiment qu'il leur revient, elles vont donc élaborer des approches pour contourner le problème.

L'ensemble des textes s'articule autour d'un même constat. Pour affirmer leur présence, les religions se rendent compte qu'elles doivent composer avec l'outil le plus puissant de la communication sociale : les médias.

28 Armando Zacarias Castillo, enseignant à l'Université de Guadalaraja (Mexique), expose la lutte qu'a menée l'Église catholique du Mexique pour contrer l'interdiction de posséder des médias d'information. Ayant réalisé le rôle important que jouaient les médias dans les autres pays latino-américains, l'Église du Mexique a revendiqué un accès direct aux médias, accès que le pouvoir civil refusait toujours de lui accorder.

Christian Mesnil, maître de conférences au Département Gestion des Entreprises et des Administrations de l'Université du Littoral, expose les stratégies de communication des protestants français et signale un double phénomène: les protestants ont de la difficulté à se trouver un créneau porteur spécifique dans les médias tant leur discours est en symbiose avec le discours social régnant. Par ailleurs, ils sont partagés entre la discrétion et la réserve que leur dicte la tradition, et l'ouverture aux médias qu'ils pratiquent de façon efficace quoique souvent plus empirique que stratégique. 
30 Hugues Hotier, professeur à l'Institut des Sciences de l'information et de la communication de l'Université Michel de Montaigne, Bordeaux, aborde le cas des télévangélistes américains qui ont dû marier la théâtralité à leur liturgie pour séduire les téléspectateurs.

31 Jean-Claude Guyot, assistant au Département de communication de l'Université catholique de Louvain, analyse les différents modes d'expression du discours religieux qui se situe tantôt dans l'espace du magistère, tantôt dans le langage de la foi, tantôt dans le discours de réappropriation de la tradition.

32 Paul Stryckman, professeur au Département d'information et de communication de l'Université Laval, Québec, élabore une approche plus conceptuelle. Dans la mesure où les églises, les dénominations et les sectes peuvent être traitées comme des organisations normatives productrices de croyances et de pratiques dans le champ religieux, ces organisations doivent nécessairement résoudre des problèmes d'orthodoxie sur le marché de la vérité symbolique. Et ce, d'autant plus que la tranmission des croyances en cette période de changements sociaux et de crise interne affecte la légitimité et l'autorité des hiérarchies religieuses.

33 Bernard Dagenais, professeur au Département d'information et de communication de l'Université Laval, Québec, pose le problème dans son principe même : non seulement les différentes églises ont appris à utiliser les médias dans leur démarche de propagation de la foi, mais elles ont aussi subi la logique des médias qui les a obligées à redéfinir leur présence sociale et leur discours.

34 François Demers, professeur au Département d'information et de communication de l'Université Laval, explique de son côté, comment la revue Ressources, un média spécialisé dans la nouvelle spiritualité, confirme les critiques habituellement adressées aux médias. L'information se réduit en valeur marchande. Il prend appui sur le drame de l'Ordre du Temple Solaire pour démontrer que la revue Ressources a modulé son discours en fonction de ses stratégies marketing.

35 Claude Cossette, professeur à l'Ecole des arts visuels de l'Université Laval, Québec, explore l'utilisation du religieux dans la publicité et démontre sa récupération dans l'univers profane du vin, du fromage et de tant d'autres produits.

36 Ces différentes approches illustrent la préoccupation pressante de la religion de s'affirmer sur la place publique et de développer des stratégies de communication adéquates pour partager l'espace médiatique avec les autres institutions.

37 BAILLARGEON Stéphane 1996, «Sondage réalisé dans le cadre du synode diocésain. Pour une Eglise terre à terre ».

38 CLELAND Kim, "Ad, promo strategies make new converts. Churches place faith in secular media, even online », Advertising age, 1995, 10 avril, p. 1 et 8.

DART John et Jimmy Allen, Bridging the gap : religion and the news media, The Freedom Forum, First Amendment Center at Venderbilt University, Nashville, 1993, 87 p.

DENNIS Everette E., « Putting more value on religion as news » Communiqué, bulletin du Freedom Forum Media Studies Center, vol. 8, n² 2, octobre, 1993, p. 2.

41 FLIPO Jean-Paul, Le marketing de l'Église, Paris, Editions du Cerf, $281 \mathrm{p}$.

42 GERBNER George et al, 1984, Religion and Television, A research report by the Annenberg of Communications (Pennsylvania) and the Gallup Organization, Résumé, avril, $14 \mathrm{p}$.

JACKSON James O., « Church and State at Cross-Purposes », Time, 28 août, 1995, p. 43. 
44 SANZ Marie, « Les E.U. replongent dans un cauchemar à peine oublié », Québec, Le Soleil, 15 juin, 1996, p. 422. SERRILL Michael S., "What the Pope Will find : John Paul II returns to a region where Catholic Marxism is out of flavor. The big threat : Evangelicals », Time, 12 février, 1966, p. 45. UPDIKE John, In the Beauty of the Lillies, Knopf, 1996, 491 p. 